\title{
Perfil de resistência de Acinetobacter baumannii a antimicrobianos em um hospital universitário de Cuiabá-MT
}

\author{
Acinetobacter baumannii resistant to antimicrobial \\ drugs from an University Hospital at Cuiabá - MT
}

Rafaella Velazquez RICAS; Thayanny do Carmo MARQUES;

Ana Caroline Akeme YAMAMOTO

Faculdade de Farmácia da Universidade de Cuiabá/UNIC. Avenida Manoel José de Arruda, 3100, Jardim Europa, Cuiabá - MT, 78065-900.

E-mail: thayannymarques@hotmail.com

\begin{abstract}
Acinetobacter baumanii has recently taken special notice, and numerous are the reports of infection breakouts in hospital environments worldwide. The extensive use of antibacterial drugs in hospitals has contributed to the increase of muti-resistant strains of A. baumanii, including resistance to carbapenems, which makes the bacteria more difficult to eliminate. This study intends to analyze the susceptibility profiles of different strains of A. baumanii isolated from clinical samples of patients in treatment in Hospital Universitário de Cuiabá - MT. It consists of a retrospective descriptive study. The bacteria were isolated in appropriate culture media. The identification and antimicrobial susceptibility testing were performed by biochemical tests and Kirby Bauer qualitative method, and Etest quantitative one. From 184 tested samples, $85.3 \%$ showed multidrug resistance, especially to cephalosporins (3rd and 4th generation), aminoglycosides and carbapenems. However, $100 \%$ of the strains were susceptible to polymyxin B. It was observed that the intensive care unit was the most affected by A. baumannii, and tracheal aspirate was the most prevalent site of infection by this pathogen.
\end{abstract}

Keywords: Acinetobacter baumannii, antimicrobials, multidrug-resistant

\section{RESUMO}

Acinetobacter baumannii vem assumindo importante papel nos processos infecciosos, e inúmeros são os relatos de surto de infecção hospitalar no mundo. O uso extensivo de terapia antimicrobiana em hospitais tem contribuído para a seleção e para o aumento no número de isolados de A. baumannii multirresistentes aos antimicrobianos, inclusive aos carbapenêmicos, dificultando o tratamento. Este estudo tem por objetivo levantar o perfil de susceptibilidade das cepas de A. baumannii isoladas de amostras clínicas de pacientes internados em um Hospital Universitário de Cuiabá -MT. Trata-se de um estudo descritivo retrospectivo. As bactérias foram isoladas em meios de cultura apropriados. A identificação e o teste de susceptibilidade aos antimicrobianos foram realizados por meio de provas bioquímicas e método qualitativo de Kirby Bauer e quantitativo Etest. Das 184 amostras avaliadas, 85,3\% apresentaram multirresistência, principalmente a cefalosporinas ( $3^{\circ}$ e $4^{\circ}$ geração), aminoglicosídeos e carbapenêmicos. No entanto, $100 \%$ das cepas foram sensíveis a polimixina B. A Unidade de Terapia Intensiva foi a mais acometida por A. baumannii, assim como aspirado traqueal foi o sítio de infecção mais prevalente por este patógeno.

Palavras Chave: Acinetobacter baumannii, antimicrobianos, resistência 


\section{INTRODUÇÃO}

Acinetobacter baumannii são cocobacilos Gram negativos não fermentadores de glicose, capazes de sobreviver por longos períodos no ambiente nosocomial, fator este que favorece sua propagação hospitalar. Acinetobacter baumannii pode estar presente no ambiente hospitalar em máquinas de diálise; nos ventiladores mecânicos; nos monitores de pressão arterial; nos sistemas de ventilação; nas fontes de água; na pele e mucosas dos profissionais de saúde e dos doentes; nas preparações medicamentosas e até nos desinfetantes. Uma ampla variedade de objetos de uso do paciente tais como cama, travesseiro, aparelho de televisão e de som, têm sido encontrados contaminados com Acinetobacter spp, podendo servir como reservatórios durante um surto nosocomial $(1,2,3,4)$.

O gênero Acinetobacter sofreu significativas modificações taxonômicas nos últimos 30 anos, sendo a espécie Acinetobacter baumannii o principal representante desse gênero. $\mathrm{O}$ microrganismo normalmente tem como alvos principais pacientes hospitalizados críticos, como os pacientes internados nas unidades de terapia intensiva (UTI) com integridade da pele e proteção das vias aéreas violadas, fatores estes que podem predispor também ao desenvolvimento de multirresistência antimicrobiana extremamente rápida. Desta forma é essencial a detecção precoce da colonização e ou infecção por este agente no meio hospitalar, para que se possam estabelecer medidas preventivas e terapêuticas adequadas. A incorreta intervenção nestas situações determinará a presença contínua deste agente nos sistemas de saúde $(2,5)$.

A endemia de $A$. baumannii nas instituições de saúde e a disseminação dos mecanismos de resistência aos carbapenêmicos resultam no aumento significativo da mortalidade, morbidade e custos, aspectos estes preocupantes relacionados a esse agente (6). Portanto, o presente estudo teve por objetivo levantar o perfil de susceptibilidade das cepas de Acinetobacter baumannii, isoladas de amostras clínicas de pacientes internados em um Hospital Universitário de Cuiabá - MT, no período de $2011-2012$.

\section{MATERIAL E MÉTODOS}

Foi realizado um estudo descritivo retrospectivo de pacientes internados com culturas positivas, para Acinetobacter baumannii laboratorialmente confirmadas, de um Hospital Universitário de Cuiabá - MT, no período de Janeiro de 2011 a Dezembro de 2012. Essas informações foram obtidas dos livros de registro do setor de microbiologia clínica do hospital avaliado. O levantamento das informa- ções sobre os microrganismos isolados ocorreu somente de forma qualitativa (gênero, espécie e perfil de susceptibilidade), não envolvendo utilização de dados pessoais de pacientes, prontuários médicos ou mesmo isolados de amostras biológicas.

Segundo protocolo do laboratório de microbiologia clínica, todas as amostras coletadas foram semeadas em placas de petri contendo meios de cultura de Agar sangue, chocolate e MacConkey para isolamento bacteriano. Após o período de incubação (18 a 24 horas), em estufa Eletrolab BOD a $35^{\circ} \mathrm{C}$ o crescimento microbiológico foi avaliado, e determinadas as características morfotintoriais pela coloração de Gram dos microrganismos isolados, quando necessário. Posteriormente foi realizada a identificação fenotípica por meio de provas bioquímicas e testes complementares para identificação de bacilos Gram negativos não fermentadores de glicose utilizando o Kit de Não Fermentadores NF II da Probac ${ }^{\circledR}$. Os isolados clínicos foram submetidos aos testes de susceptibilidade aos antimicrobianos pelo método de Kirby Bauer e método quantitativo (Etest - Probac $\left.{ }^{\circledR}\right)$ de acordo com a padronização da CLSI do ano vigente.

\section{RESULTADOS E DISCUSSÃO}

No período de Janeiro de 2011 a Dezembro de 2012 foram isoladas 184 culturas positivas para bactéria A. baumannii provenientes de pacientes internados no Hospital Universitário avaliado.

Figura 1. Distribuição das cepas de Acinetobacter baumannii nas unidades de internação de um Hospital Universitário em Cuiabá - MT, no período de 2011-2012.

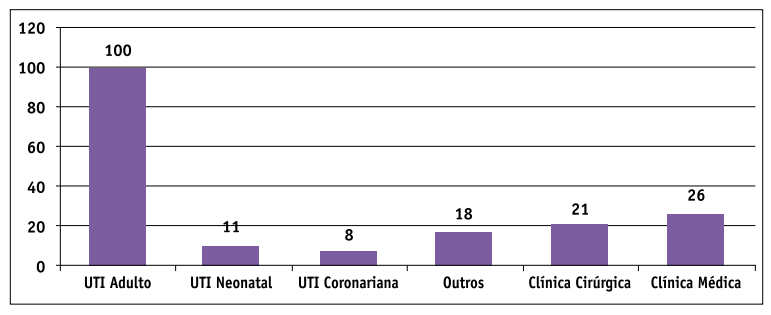

Das unidades de internação analisadas, as unidades de terapia intensiva (UTI) apresentaram mais casos de infecção por $A$. baumannii, totalizando 119 casos $(64,7 \%)$, com destaque para a UTI adulto que isoladamente foi responsável por 100 dos 184 casos de infecção por A. baumannii, conforme Figura 1.

A Figura 2 mostra a prevalência dos sítios de infecção analisados neste estudo, que foram: aspirado traqueal, ponta de cateter, sangue (hemocultura), urina 
(urocultura), secreções (cirúrgicas, mielomeningocele, tumor, abscesso) e outros (líquor, tecido, escara, etc), sendo que o sitio de infecção mais prevalente foi aspirado traqueal 63 (35\%), seguido de secreções e urocultura $31(17 \%)$.

Figura 2. Prevalência de $A$. baumannii em sítio de infecções em um Hospital Universitário em Cuiabá - MT, no período de 2011-2012.

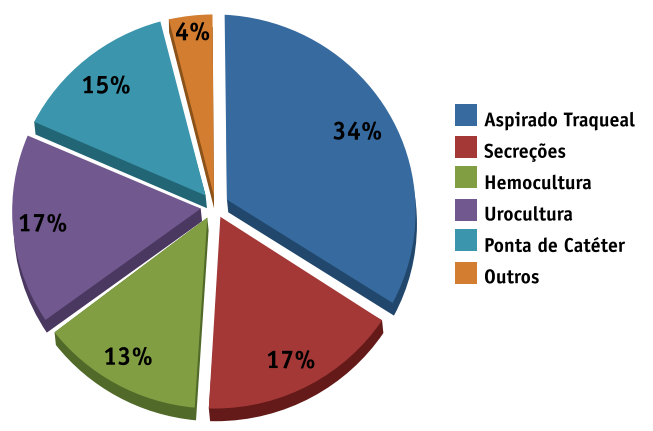

Com relação ao perfil de susceptibilidade aos antimicrobianos (Figura 3), das 184 cepas de Acinetobacter baumanni analisadas, 157 (85,3\%) apresentaram multirresistência. Cefepime, uma cefalosporina de $4^{\circ}$ geração, não foi eficaz em 149 (81\%) dos casos; as cefalosporinas de $3^{\circ}$ geração ceftazidima e ceftriaxona, em $154(83,7 \%)$ e $152(82,6 \%)$, respectivamente. O aminoglicosídeo amicacina não mostrou atrividade em 124 (67,4\%) dos casos. Com relação aos antibióticos carbapenêmicos,a resistência do patógeno a imipenem foi observada em 133 casos $(72,3 \%)$ e para meropenem em 139 casos (75,5\%).

No entanto, as 184 cepas de Acinetobacter baumanni (100\%) foram sensíveis a polimixina B e 100 $(54,3 \%)$ cepas foram sensíveis a ampicilina + sulbactama.

\section{DISCUSSÃO}

A importância do Acinetobacter baumannii tem aumentado nos últimos anos devido à sua grande capacidade em adquirir mecanismos de resistência às diferentes classes de antibióticos e à sua grande aptidão em sobreviver e se adaptar a condições adversas. Todos estes fatores tornam-no responsável por uma morbidade e mortalidade elevada, especialmente, nos doentes críticos $(7,8)$. A Acinetobacter baumannii é uma das bactérias que consegue sobreviver por mais tempo fora do seu habitat, sendo um grande problema de infecções graves e resistência rápida a antimicrobianos (9).

O perfil de resistência da $A$. baumannii a antimicrobianos varia muito de país para país e até de setores de um mesmo hospital. (10). Neste estudo pode-se observar que os pacientes internados nas UTIs foram muito mais acometidos por infecção por A. baumannii, com 119 casos $(64,7 \%)$, enquanto os demais pacientes internados totalizando 65 casos $(35,3 \%)$. Este resultado era esperado, uma vez que a unidade de terapia intensiva concentra os pacientes mais graves da clientela hospitalar, que apresentam doenças ou condições clínicas predisponentes a infecções, são submetidos a procedimentos invasivos ou imunossupressivos e com maior utilização de antimicrobianos que podem levar a resistência $(9,11)$.

Acinetobacter baumannii é a principal espécie do gênero Acinetobacter, e manifestou-se como potente patógeno de infecções de âmbito hospitalar. É considerado um grande problema em infecções pulmonares (9). Neste estudo, $34 \%$ dos casos de infecção por $A$. baumannii eram provenientes de amostras respiratórias - aspirado traqueal, corroborando com o estudo de Pontes e cols (2006) no Hospital Geral de Fortaleza, onde o sítio de infecção em que foi isolada a maioria das cepas de $A$. baumannii foi aspirado traqueal $39 \%$ (5), assim como no estudo de Wu e cols (2002), em que a frequência deste mesmo patógeno foi de $27 \%$, sendo todas as amostras de aspirado traqueal (12).

Figura 3. Perfil de susceptibilidade aos antimicrobianos de Acinetobacter baumannii em um Hospital Universitário em Cuiabá - MT, no período de 2011 - 2012

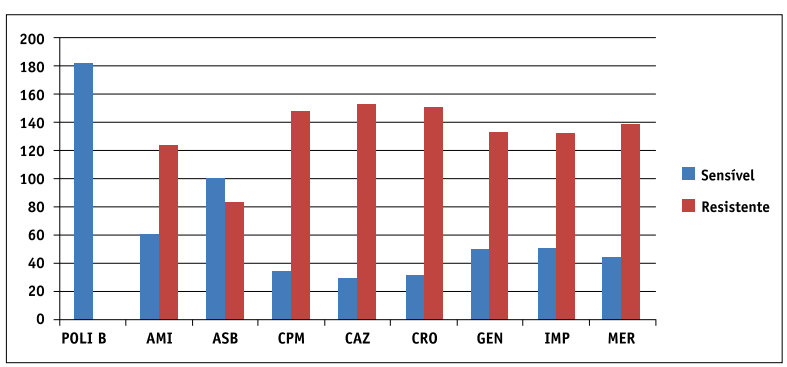

POLI B (polimixina B); AMI (amicacina); ASB (ampicilina+sulbactam); CPM (cefepime); CAZ (ceftazidima); CRO (ceftriaxona); GEN (gentamicina); IMP (imipenem); MER (meropenem).

A multirresistência de Acinetobacter baumannii é um problema em muitos hospitais. Os antibióticos carbapenêmicos exerciam um papel importante no manejo de infecções severas causadas por esse patógeno. No entanto, o aparecimento de cepas resistentes aos carbapenêmicos é cada vez mais frequente devido à produção de enzimas beta-lactamases pertencentes às classes $\mathrm{D}$ e $\mathrm{B}$ de Ambler, as metalo-beta-lactamases (MBLs) e a produção de carbapenemases do tipo OXA $(13,14)$. Neste estudo, das 184 cepas de Acinetobacter baumanni analisadas ,157 (85,3\%) apresentaram multirresistência. Maltezou (2009) observou que cepas produtoras de carbapenema- 
ses apresentavam fenótipos de multirresistência (15). Foram evidenciados elevados níveis de resistência aos antibióticos carbapenêmicos (imipenem e meropenem) em 133 casos (72,3\%) e 139 casos $(75,5 \%)$, respectivamente, além de resistência a cefepime em 149 (81\%) dos casos, ceftazidima $154(83,7 \%)$, ceftriaxona $152(82,6 \%)$ e a amicacina em $124(67,4 \%)$. Estes dados corroboram o estudo de Soares \& Carvalho (2011), que encontrou cepas apresentando altas taxas de resistência aos outros antimicrobianos testados, como o cefepime (97,7\%), ceftazidime $(88,6 \%)$, amicacina $(81,8 \%)$ (16). Desta forma a emergência de resistência entre Acinetobacter baumannii é preocupante, deixando poucas opções terapêuticas no tratamento de infecções por estes patógenos.

Por outro lado, as 184 cepas de Acinetobacter baumannii (100\%) foram sensíveis a polimixina B e 100 $(54,3 \%)$ cepas foram sensíveis a ampicilina+sulbactama. A polimixina B é seletivamente tóxica a bactérias Gram-negativas devido à sua especificidade pelas moléculas de lipopolissacarídeo (8). As polimixinas têm sido efetivas no tratamento de infecções por A. baumannii, com pouco desenvolvimento de resistência, mas a nefrotoxicidade limita seu uso. Esses fármacos se acumulam nos tecidos, especialmente rins e cérebro, mas o meca- nismo molecular de toxicidade ainda não é claro. Uma opção terapêutica é o sulbactama, que é um inibidor de $\beta$-lactamase para ser usado junto a outros antimicrobianos $\beta$-lactâmicos, tais como ampicilina. A maioria das espécies de Acinetobacter baumannii permanece suscetível a ampicilina+sulbactama. Estudos no Brasil e na Itália confirmam a elevada taxa de suscetibilidade de Acinetobacter spp a ampicilina+sulbactama (1).

\section{CONCLUSÃO}

Dos 184 casos de infecção por Acinetobacter baumannii avaliados neste estudo, 157 ou $85,3 \%$ eram cepas multirresistentes, principalmente a antibióticos amplamente utilizados na prática clínica, tais como cefalosporinas de $3^{\circ}$ e $4^{\circ}$ geração, aminoglicosídeos e carbapenêmicos. As cepas apresentaram sensibilidade em sua grande maioria aos antibióticos polimixina B (100\%) e ampicilina+sulbactam (54,3\%), restando pouca alternativa terapêutica para tratamento dessas infecções, cada vez mais frequentes no ambiente hospitalar, principalmente em amostras respiratórias como no aspirado traqueal e nas unidades de terapia intensiva, conforme constatado no presente estudo.

\section{REFERÊNCIAS}

1. Luiz SO. Caracterização da resistência de amostras de Acinetobacter Baumannii isoladas no Hospital de Clínicas de Curitiba. [Tese]. Curitiba: Faculdade de Ciências Biológicas, Universidade Federal do Paraná. 2006.

2. Peleg AY, Seifert H, Paterson DL. Acinetobacter baumannii: Emergence of a Successful Pathogen. Clin Microbiol Rev. 2008. 21(3): 538-582.

3. Silva RNP. A Importância do Acinetobacter baumannii na infecção Adquirida nos Cuidados de Saúde. [Tese]. Instituto de Ciência Biomédicas de Abel Salazar - Porto, Universidade do Porto. 2009.

4. Oliveira AC, Damasceno QS. Superfícies do ambiente hospitalar como possíveis reservatórios de bactérias resistentes: uma revisão. Rev Esc Enferm USP. 2010. 44(4):118-23.

5. Pontes VMO; Menezes EA, Cunha FA, Ângelo MRF, Salviano MNC, Oliveira IRN. Perfil de Resistência de Acinetobacter baumannii a Antimicrobianos nas Unidades de Terapia Intensiva e Semi-Intensiva do Hospital Geral de Fortaleza. RBAC. 2006. 38(2): 123-6.

6. Fernandes AT, Fernandes MOV, Filho NR. Infecção Hospitalar e suas interfaces na área da saúde. São Paulo: Atheneu. 2000.

7. Gaynes R; Edward JR. Overview of nosocomial infections caused by gram-negative bacilli. Clin Infect Dis. 2005. 41(6):848-54.

8. Falagas ME, Bliziotis IA, Siempos II. Attributable mortality of Acinetobacter baumannii infections in critically ill patients: a systematic review of matched cohort and case-control studies. Crit Care. 2006.10(2):R48.
9. Cisneros JM, Bãno JR. Nosocomial bacteremia due to Acinetobacter baumannii: epidemiology, clinical features and treatment. Clin Microbiol Infect. 2002. 8(11):687-93.

10. Bergogne-Bérézin E, Towner KJ. Acinetobacter spp. as nosocomial pathogens: microbiological, clinical and epidemiological features. Clin Microbiol Rev. 1996. 9(2): 148-65.

11. Zanon U, Neves J. Infecções hospitalares: prevenção, diagnóstico e tratamento. Rio de Janeiro: Medsi; 1987.

12. Wu CL, Yang DI, Wang NY, Kuo HT, Chen PZ. Quantitative culture of endotracheal aspirates in the diagnosis of ventilator-associated pneumonia in patients with treatment failure. Chest. 2002. 122 (2): 662-8.

13. Mendes RE, Castanheira M, Pignatari AC, Gales AC. Metalo-beta-lactamases. J Brás Patol Med Lab. 2006. 42(2):103-13.

14. Uma KR, Srinivasa RR, Sahoo S, Shashikala P, Kanungo R, Jayachandran S, Prashanth K. Phenotypic and genotypic assays for detecting the prevalence of metallo-beta-lactamases in clinical isolates of Acinetobacter baumannii from South Indian tertiary care hospital. J Med Microbiol. 2009. 58(4):430-5.

15. Maltezou HC. Metallo-beta-lactamases in Gram-negative bacteria: introducing the era of pan-resistance? Int $\mathbf{J}$ Antimicrob Agents. 2009. 33(5):405.e 1-7.

16. Soares VM, Carvalho GCD. Atividade in vitro da tigeciclina frente aos isolados de Acinetobacter baumannii no Hospital Júlia Kubitschek da Fundação Hospitalar de Minas Gerais. RBAC. 2011. 43(3):197-200. 